\title{
Non-valvular atrial fibrillation: impact of apixaban on patient outcomes
}

\author{
This article was published in the following Dove Press journal: \\ Patient Related Outcome Measures \\ 3 November 2017 \\ Number of times this article has been viewed
}

\author{
Adam loannou' \\ Irene Tsappa ${ }^{2}$ \\ Sofia Metaxa ${ }^{3}$ \\ Constantinos G Missouris ${ }^{2,3}$ \\ 'Cardiology Department, Royal Free \\ NHS Foundation Trust, London, UK; \\ ${ }^{2}$ Cardiology Department, Medical \\ School, University of Cyprus, Nicosia, \\ Cyprus; ${ }^{3}$ Cardiology Department, \\ Frimley Health NHS Foundation Trust, \\ London, UK
}

\begin{abstract}
Atrial fibrillation is the most common arrhythmia worldwide, and carries a significantly increased risk of thromboembolic stroke. Initially, vitamin $\mathrm{K}$ antagonists were used as stroke prophylaxis; but more recently, a group of drugs known as novel oral anticoagulants have been developed. Apixaban belongs to this group of drugs, and is a factor Xa inhibitor that has emerged as a popular pharmacological agent worldwide. In this review, we will provide an overview of the pivotal trials in the development of apixaban, while also critically evaluating the new emerging real-world data, and discussing the effectiveness, safety, economic viability and future prospects of apixaban and how it impacts on patient outcomes in those with nonvalvular atrial fibrillation.
\end{abstract}

Keywords: apixaban, atrial fibrillation, warfarin, stroke, bleeding

\section{Introduction}

Atrial fibrillation (AF) is the most common arrhythmia occurring in $0.1 \%-4.0 \%$ of the population and the prevalence rising to $7.2 \%$ in patients aged $\geq 65$, with a yearly increase in incidence of $1.6 \%$ in patients aged $\geq 75$. AF is a major risk factor for ischemic stroke, secondary to cardiac emboli that commonly form in the left atrial appendage as a result of blood stasis, and these emboli result in stroke that is commonly more disabling than stroke from other causes. ${ }^{1-3}$

A number of clinical trials have confirmed that the use of vitamin $\mathrm{K}$ antagonists (VKAs), such as warfarin, as a form of anticoagulation significantly reduces the risk of stroke in patients with AF. However VKAs do have a slow onset of action, narrow therapeutic index and multiple drug interactions, all of which contribute to a requirement for regular anticoagulation monitoring and dose adjustment.

Furthermore, they are effective when the anticoagulation as assessed by the international normalized ratio (INR) is within $2-3$; it is generally accepted that a time in the therapeutic range (TTR) $>70 \%$ is required for adequate anticoagulation, and those with poor control are at a higher risk of either major bleeding or a severe/fatal thromboembolic event. ${ }^{4,5}$

A new group of oral anticoagulant agents known as novel oral anticoagulants, which, more recently, have been renamed as direct oral anticoagulants (DOACs), have been developed in an attempt to overcome the drawbacks seen with warfarin. Apixaban belongs to this class of drugs and is a direct oral factor Xa inhibitor, with rapid absorption, 50\% bioavailability and a 12-hour half-life, meaning it requires a twicedaily dosing regimen. The use of apixaban negates the need for regular monitoring
Correspondence: Constantinos G Missouris Frimley Health NHS Foundation Trust, Wexham Street, Slough SL2 4HL, UK Tel +442070794217

Fax +44207580 0122

Email dinos.missouris@fhft.nhs.uk 
of anticoagulation levels, through INR measurements, but due to its $25 \%$ renal excretion, annual monitoring of renal function is recommended. ${ }^{6}$

\section{Pivotal AVERROES and ARISTOTLE trials}

Up until relatively recently, VKAs were the gold standard treatment for stroke prevention in patients with AF. However, due to the aforementioned disadvantages, many patients were deemed unsuitable for treatment, and were left with inferior antiplatelet agents, such as aspirin and/or clopidogrel. Although antiplatelet agents reduce the risk of stroke by up to $20 \%$ in patients with AF, their therapy is still vastly inferior to the substantially more efficacious warfarin. ${ }^{7}$ Growing concerns were expressed amongst clinicians that those unsuitable for warfarin therapy were being exposed to a greater risk of thromboembolic stroke.

The Apixaban Versus Acetylsalicylic Acid to Prevent Stroke in AF Patients Who Have Failed or Are Unsuitable for Vitamin K Antagonist Treatment (AVERROES) trial was designed to determine the efficacy and safety of apixaban (5 mg bd), compared with aspirin (81-324 mg daily) in the treatment of patients with AF, for whom VKA therapy was considered unsuitable. After a mean duration of 1.1 years follow-up, the study was terminated early due to the overwhelming success of apixaban. The trial concluded that apixaban reduced the rate of ischemic stroke (1.1\% per year vs $3.0 \%$ per year; hazard ratio $\mathrm{HR}=0.37$; $95 \% \mathrm{CI}=0.25-0.55$; $P<0.001)$ and the rate of hospitalization for cardiovascular disease $(12.6 \%$ per year vs $15.9 \%$ per year; $H R=0.79 ; 95 \%$ $\mathrm{CI}=0.69-0.91 ; P<0.001)$, without significantly increasing the incidence of major bleeding (1.4\% per year vs $1.2 \%$ per year; $\mathrm{HR}=1.13 ; 95 \% \mathrm{CI}=0.74-1.75 ; P=0.57$ ) or intracranial hemorrhage $(0.4 \%$ per year vs $0.4 \%$ per year; $\mathrm{HR}=0.85 ; 95 \%$ $\mathrm{CI}=0.74-1.75 ; P=0.57){ }^{8}$

The Apixaban for Reduction In Stroke and other ThromboemboLic Events in AF (ARISTOTLE) trial was the first large randomized controlled trial that directly compared the efficacy of apixaban to warfarin. This double blind trial compared apixaban ( $5 \mathrm{mg}$ bd) with warfarin (target INR of 2.0-3.0) in 18,201 patients with non-valvular AF (NVAF). During a median follow-up duration of 1.8 years, this study concluded that apixaban was superior to warfarin in preventing stroke/systemic emboli ( $1.27 \%$ vs $1.60 \%$; $\mathrm{HR}=0.79 ; 95 \%$ $\mathrm{CI}=0.66-0.95 ; P<0.001$ for non-inferiority and $P=0.01$ for superiority), causing less major bleeding $(2.13 \%$ vs $3.09 \%$; $\mathrm{HR}=0.69 ; 95 \% \mathrm{CI}=0.60-0.80 ; P<0.001)$, and a lower mortality rate $(3.52 \%$ vs $3.94 \%$; $\mathrm{HR}=0.89 ; 95 \% \mathrm{CI}=0.80-0.99$; $P=0.047){ }^{9}$
There have been multiple post hoc analysis studies of the ARISTOTLE trial population, which evaluated the outcomes of various sub-groups of patients. They demonstrated that apixaban produces similar outcomes in patients with previous stroke/transient ischemic attack (TIA), ${ }^{10}$ heart failure, ${ }^{11}$ and coronary artery disease, ${ }^{12}$ but reduced bleeding events in those with peripheral artery disease, ${ }^{13}$ renal dysfunction, ${ }^{14}$ diabetes mellitus ${ }^{15}$ and polypharmacy. ${ }^{16}$ Apixaban was associated with lower rates of myocardial infarction in patients with hypertension ${ }^{17}$ and reduced rates of intracranial hemorrhage in those who were previously on warfarin. Its effectiveness was not modified by previous use of VKAs, indicating patients could be safely switched from warfarin to apixaban, and benefit from overall improved outcomes. ${ }^{18}$ (Table 1)

\section{Real-world data regarding bleeding risk}

Since the success of multiple clinical trials in demonstrating the safety and efficacy of apixaban, real-world data have begun to emerge.

Recently, a large observational study evaluated the efficacy of apixaban in 2514 patients, with a diagnosis of NVAF and a history of ischemic stroke/TIA and concluded that apixaban was no worse than warfarin with regards to the incidence of ischemic stroke, intracranial hemorrhage or major bleeding. This confirmed the safety profile documented in the respective Phase III trials, but failed to demonstrate the reduction in ischemic stroke that had previously been proven. ${ }^{19}$

Interestingly, a large systematic review of 26 studies carried out between 2012 and 2016 not only confirmed the safety profile of apixaban, but went as far as to state that its use was consistently associated with a lower risk of major bleeding than warfarin. The same could not be said for dabigatran, which yielded far less consistent results, or rivaroxaban, which had a similar bleeding risk to warfarin. The study concluded that among the DOACs reviewed (apixaban, dabigatran and rivaroxaban), the risk of major bleeding was significantly lower with apixaban than rivaroxaban, but similar to dabigatran, despite its more consistent profile. ${ }^{20}$

These results were echoed in a further propensitymatched real-world analysis that recruited 45,361 newly anticoagulated patients with NVAF, 7438 (16.4\%) of whom were taking apixaban. This study showed that apixaban had a significantly lower risk of major bleeding than warfarin (major bleeding incidence rate per 100 person years 2.38 vs $4.46 ; \mathrm{HR}=0.53 ; 95 \% \mathrm{CI}=0.39-0.71)$, dabigatran $(2.24 \mathrm{vs}$ $3.02 ; \mathrm{HR}=1.41 ; 95 \% \mathrm{CI}=0.93-2.14)$ and rivaroxaban $(2.24$ vs $4.24 ; \mathrm{HR}=1.82 ; 95 \% \mathrm{CI}=1.36-2.43)$. These differences 
Table I Summary of ARISTOTLE trial post-hoc analysis

\begin{tabular}{|c|c|c|c|c|c|c|}
\hline $\begin{array}{l}\text { Study and subset of } \\
\text { patients }\end{array}$ & $\begin{array}{l}\text { Number of } \\
\text { participants }\end{array}$ & Outcomes & $\begin{array}{l}\text { Apixaban } \\
\text { rate } \\
(100 \mathrm{py})\end{array}$ & $\begin{array}{l}\text { Warfarin } \\
\text { rate } \\
(100 \text { py })\end{array}$ & $\begin{array}{l}\text { Hazard ratio } \\
(95 \% \mathrm{Cl})\end{array}$ & $P$-value \\
\hline Easton et al ${ }^{10}$ & Stroke/TIA n=3436 & Stroke/systemic embolism & 2.46 & 3.24 & $0.76(0.56-1.03)$ & 0.71 \\
\hline Patients with previous & & All-cause mortality & 4.22 & 4.77 & $0.89(0.70-1.12)$ & 0.89 \\
\hline \multirow[t]{2}{*}{ stroke/TIA } & & Total bleeding & 19.86 & 29.12 & $0.70(0.62-0.79)$ & 0.70 \\
\hline & & Major bleeding & 2.84 & 3.91 & $0.73(0.55-0.98)$ & 0.69 \\
\hline McMurray et al" & LVSD n=2736 (19\%) & Stroke/systemic embolism & & & & \\
\hline Patients with both HF & & LVSD & 0.99 & 1.80 & $0.55(0.34-0.91)$ & 0.21 \\
\hline status and LV systolic & HF-PEF $n=3207$ & HF-PEF & 1.51 & 1.54 & $0.98(0.65-1.49)$ & \\
\hline \multirow[t]{9}{*}{ function } & $(22 \%)$ & No LVSD/No HF & 1.16 & 1.58 & $0.74(0.57-0.96)$ & \\
\hline & No LVSD/No HF & All-cause mortality & & & & \\
\hline & $\mathrm{n}=8728(59 \%)$ & LVSD & 6.99 & 7.15 & $0.98(0.79-1.21)$ & 0.52 \\
\hline & & HF-PEF & 4.05 & 4.58 & $0.89(0.69-1.13)$ & \\
\hline & & No LVSD/No HF & 2.17 & 2.62 & $0.83(0.68-1.01)$ & \\
\hline & & Major bleeding (ISTH) & & & & \\
\hline & & LVSD & 2.77 & 3.41 & $0.81(0.58-1.14)$ & 0.50 \\
\hline & & HF-PEF & 1.95 & 3.17 & $0.62(0.44-0.88)$ & \\
\hline & & No LVSD/No HF & 2.17 & 2.83 & $0.77(0.62-0.94)$ & \\
\hline Bahit et al ${ }^{12}$ & CAD $n=6639$ & Stroke/systemic embolism & 1.47 & 1.55 & $0.950(0.7 \mid 2-1.267)$ & 0.11 \\
\hline Patients with prior & & All-cause mortality & 4.21 & 4.40 & $0.958(0.809-1.133)$ & 0.28 \\
\hline CAD & & Myocardial infarction & 0.95 & 1.00 & $0.947(0.662-1.354)$ & 0.45 \\
\hline Hu et $a^{13}$ & PAD $n=884$ & Stroke/systemic embolism & 1.79 & 2.56 & $0.63(0.32-1.25)$ & 0.52 \\
\hline Patients with PAD at & & Myocardial infarction & 1.14 & 1.26 & $0.97(0.39-2.38)$ & 0.87 \\
\hline baseline & & Clinically relevant bleeding & 6.95 & 6.75 & $1.05(0.69-1.58)$ & 0.03 \\
\hline Hohnloser et al ${ }^{14}$ & eGFR $>80 n=7518$ & Stroke/systemic embolism & & & & \\
\hline Patients divided & $(42 \%)$ & $>80$ & 0.99 & 1.12 & $0.88(0.64-1.22)$ & 0.705 \\
\hline according to eGFR & $>50-80$ & $>50-80$ & 1.24 & 1.69 & $0.74(0.56-0.97)$ & \\
\hline by Cockroft-Gault & $n=7587(42 \%)$ & $\leq 50$ & 2.11 & 2.67 & $0.79(0.55-1.14)$ & \\
\hline \multirow[t]{8}{*}{$\mathrm{mL} / \mathrm{min}$} & & All-cause mortality & & & & \\
\hline & $\leq 50 n=3017(15 \%)$ & $>80$ & 2.33 & 2.71 & $0.86(0.70-1.06)$ & 0.627 \\
\hline & & $>50-80$ & 3.41 & 3.56 & $0.96(0.81-1.14)$ & \\
\hline & & $\leq 50$ & 7.12 & 8.30 & $0.86(0.70-1.05)$ & \\
\hline & & Major bleeding & & & & \\
\hline & & $>80$ & 1.46 & 1.84 & $0.80(0.61-1.04)$ & 0.030 \\
\hline & & $>50-80$ & 2.45 & 3.21 & $0.77(0.62-0.94)$ & \\
\hline & & $\leq 50$ & 3.21 & 6.44 & $0.50(0.38-0.66)$ & \\
\hline Ezekowitz et al ${ }^{15}$ & Diabetes mellitus & Stroke/systemic embolism & 1.39 & 1.86 & $0.746(0.529-1.053)$ & 0.7064 \\
\hline Patients with diabetes & $n=4547$ & All-cause mortality & 1.88 & 2.12 & $0.888(0.655-1.203)$ & 0.9995 \\
\hline mellitus & & ISTH major bleeding & 3.01 & 3.13 & $0.96 \mid(0.740-1.247)$ & 0.0034 \\
\hline Jaspers Focks et al ${ }^{16}$ & $0-5$ drugs $n=6943$ & Stroke/systemic embolism & & & & \\
\hline Patients with & & $0-5$ & 1.19 & 1.39 & $0.86(0.63-1.17)$ & 0.82 \\
\hline concomitant drug & $6-8$ drugs $n=6502$ & $6-8$ & 1.29 & 1.69 & $0.76(0.57-1.03)$ & \\
\hline \multirow[t]{9}{*}{ treatments } & & $\geq 9$ & 1.35 & 1.79 & $0.76(0.54-1.07)$ & \\
\hline & $\geq 9$ drugs $n=4756$ & All-cause mortality & & & & \\
\hline & & $0-5$ & 2.78 & 3.24 & $0.86(0.70-1.05)$ & 0.81 \\
\hline & & $6-8$ & 3.57 & 4.04 & $0.89(0.74-1.06)$ & \\
\hline & & $\geq 9$ & 4.55 & 4.85 & $0.94(0.77-1.14)$ & \\
\hline & & Major bleeding & & & & \\
\hline & & $0-5$ & 1.27 & 2.55 & $0.50(0.38-0.66)$ & 0.017 \\
\hline & & $6-8$ & 2.06 & 2.88 & $0.72(0.56-0.91)$ & \\
\hline & & $\geq 9$ & 3.55 & 4.21 & $0.84(0.67-1.06)$ & \\
\hline Rao et $\mathrm{al}^{17}$ & Hypertension & Stroke/systemic embolism & 1.31 & 1.59 & $0.82(0.68-0.10)$ & 0.27 \\
\hline Patients with history & $n=15,916$ & All-cause mortality & 3.38 & 3.77 & $0.90(0.79-1.01)$ & 0.96 \\
\hline \multirow[t]{2}{*}{ of hypertension } & & Myocardial infarction & 0.51 & 0.66 & $0.78(0.57-1.05)$ & 0.02 \\
\hline & & Any bleeding & 17.91 & 25.76 & $0.71(0.67-0.75)$ & 0.55 \\
\hline
\end{tabular}


Table I (Continued)

\begin{tabular}{|c|c|c|c|c|c|c|}
\hline $\begin{array}{l}\text { Study and subset of } \\
\text { patients }\end{array}$ & $\begin{array}{l}\text { Number of } \\
\text { participants }\end{array}$ & Outcomes & $\begin{array}{l}\text { Apixaban } \\
\text { rate } \\
(100 \text { py })\end{array}$ & $\begin{array}{l}\text { Warfarin } \\
\text { rate } \\
(100 \mathrm{py})\end{array}$ & $\begin{array}{l}\text { Hazard ratio } \\
(95 \% \mathrm{Cl})\end{array}$ & $P$-value \\
\hline Garcia et $\mathrm{al}^{18}$ & VKA naïve $\mathrm{n}=7800$ & Stroke/Systemic embolism & & & & \\
\hline Patients divided & & Naïve & 1.52 & 1.77 & $0.86(0.67-1.11)$ & 0.39 \\
\hline according to prior & VKA experienced & Experienced & 1.07 & $\mathrm{I} .47$ & $0.73(0.57-0.95)$ & \\
\hline \multirow[t]{9}{*}{ Warfarin use } & $n=|0,40|$ & Major bleeding & & & & \\
\hline & & Naïve & 2.17 & 2.96 & $0.73(0.59-0.91)$ & 0.50 \\
\hline & & Experienced & 2.11 & 3.18 & $0.66(0.55-0.80)$ & \\
\hline & & Intracranial bleeding & & & & \\
\hline & & Naïve & 0.48 & 0.81 & $0.60(0.38-0.93)$ & 0.02 \\
\hline & & Experienced & 0.23 & 0.80 & $0.28(0.17-0.46)$ & \\
\hline & & All-cause mortality & & & & \\
\hline & & Naïve & 4.07 & 4.50 & $0.91(0.78-1.06)$ & 0.82 \\
\hline & & Experienced & 3.10 & 3.51 & $0.88(0.76-1.03)$ & \\
\hline
\end{tabular}

Abbreviations: 100 py, event rate per 100 person years; CAD, coronary artery disease; EF, ejection fraction; HF, heart failure; ISTH, International Society on Thrombosis and Hemostasis; LV, left ventricular; LVSD, left ventricular systolic function; PAD, peripheral artery disease; PEF, preserved ejection fraction; TIA, transient ischemic attack; VKA, vitamin $\mathrm{K}$ antagonists.

were evident in spite of balanced HAS-BLED (hypertension, abnormal renal/liver function, stroke, bleeding history or predisposition, labile INR, elderly ( $>65$ years old), drugs/ alcohol misuse) scores across all cohorts. ${ }^{21}$

A large meta-analysis of 20 randomized controlled trials of 91,671 patients with AF further demonstrated the superior safety profile of standard-dose apixaban $(5 \mathrm{mg} \mathrm{bd})$ by comparing the incidence of bleeding complications with the other DOACs (dabigatran, edoxaban and rivaroxaban), warfarin and dual antiplatelet therapy (aspirin and clopidogrel). The study concluded that apixaban was associated with the lowest risk of both gastrointestinal bleeding and intracranial hemorrhage. ${ }^{22}$

\section{Real-world data regarding stroke prevention}

Along with the impressive safety profile of apixaban, demonstrated by its overall lower risk of major bleeding and intracranial hemorrhage, it has also been associated with impressive rates of stroke prevention. This was first established in various Phase II/III clinical trials, but is now being reaffirmed in studies evaluating real-world data.

A large USA database was used to identify patients with NVAF, anticoagulated between October 2010 and June 2015 for stoke prevention. Three propensity-matched cohorts were generated to compare three DOACs (apixaban, dabigatran and rivaroxaban) with warfarin, and confirmed the abovementioned findings (Table 2). Interestingly standard-dose apixaban was associated with lower risk of major bleeding compared with warfarin (event rate per 100 person years, 1.85 vs $4.58 ; \mathrm{HR}=0.38 ; 95 \% \mathrm{CI}=0.28-0.53 ; P<0.001$ ), whereas reduced-dose apixaban (2.5 $\mathrm{mg}$ bd) was associated with a similar risk of major bleeding (event rate per 100 person years, 4.53 vs 3.95 ; $\mathrm{HR}=0.74 ; 95 \% \mathrm{CI}=0.44-1.25$ ). Dabigatran was associated with lower risks of major bleeding and intracranial bleeding than warfarin, but not gastrointestinal bleeding; and rivaroxaban was associated with a higher risk of gastrointestinal bleeding, a similar risk of major bleeding, but a lower risk of intracranial bleeding compared to warfarin. ${ }^{23}$

The superiority of apixaban was also visible when effectiveness outcomes were analyzed, as it was the only DOAC associated with a reduced risk of stroke. Interestingly this reduction was driven mainly by lower rates of hemorrhagic stroke, (Table 2) while both dabigatran and rivaroxaban were associated with a similar risk of stroke/systemic embolism when compared to warfarin. ${ }^{23}$ These results were echoed in a similar propensity-matched study (Table 2) $\cdot{ }^{24}$ It could be hypothesized that the reduced bleeding risk linked to apixaban may also contribute to its associated lower risk of hemorrhagic stroke, however, further research would be needed to verify such a statement.

A large meta-analysis not only confirmed the superiority of apixaban to warfarin, but also demonstrated its superiority when compared with both aspirin alone, and aspirin and clopidogrel by significantly reducing rates of all strokes, ischemic strokes and major bleeding. ${ }^{25}$

Interestingly, dabigatran was superior to warfarin in reducing ischemic strokes, but only apixaban showed a borderline reduction in overall mortality over warfarin $(\mathrm{HR}=0.9$; $95 \% \mathrm{CI}=0.81-1.00$ ). Given that apixaban was associated with a lower risk of major bleeding than warfarin, and dabigatran was not, these results may be an indication that overall 
Table 2 Summary of real-world studies and meta-analysis comparing apixaban to warfarin

\begin{tabular}{|c|c|c|c|}
\hline Study & Number of participants & Outcomes & Hazard ratio $(95 \% \mathrm{Cl})$ \\
\hline Yoa et $\mathrm{al}^{23}$ & 15,390 & Any bleeding & $0.45(0.34-0.59)$ \\
\hline Effectiveness and safety of apixaban compared & & Intracranial hemorrhage & $0.24(0.12-0.50)$ \\
\hline \multirow[t]{4}{*}{ to warfarin based in the USA } & & Gastrointestinal bleeding & $0.5 \mathrm{I}(0.37-0.70)$ \\
\hline & & Stroke/systemic embolism & $0.67(0.46-0.98)$ \\
\hline & & Hemorrhagic stroke & $0.35(0.14-0.88)$ \\
\hline & & Ischemic stroke & $0.83(0.53-1.29)$ \\
\hline Amin et $\mathrm{a}^{24}$ & 186,132 & Stroke/systemic embolism & $0.40(0.3 \mathrm{I}-0.53)$ \\
\hline $\begin{array}{l}\text { Effectiveness and safety of apixaban compared } \\
\text { to warfarin in patients } \geq 65 \text { years old }\end{array}$ & & Major bleeding & $0.5 \mathrm{I}(0.44-0.58)$ \\
\hline Tawfik et $\mathrm{al}^{25}$ & 96,826 & All strokes & $0.79(0.66-0.96)$ \\
\hline Meta-analysis of 16 randomized controlled & & Major bleeding & $0.69(0.60-0.80)$ \\
\hline \multirow[t]{3}{*}{ trials comparing apixaban to warfarin } & & Intracranial hemorrhage & $0.42(0.30-0.57)$ \\
\hline & & Ischemic stroke & $0.96(0.77-1.20)$ \\
\hline & & Overall mortality & $0.90(0.8 \mathrm{I}-1.00)$ \\
\hline
\end{tabular}

mortality in these patients is driven more by major bleeding events than ischemic stroke. ${ }^{25}$

Apixaban was once again associated with a reduced rate of all strokes, but not a reduced rate of ischemic stroke, which further supports the hypothesis that its improved safety profile significantly reduces the risk of hemorrhagic stroke compared with its counterparts.

However, reduced rates of stroke were not observed in those with an overall lower risk of stroke. A Danish nationwide observational cohort study $(n=14,020)$ sought to compare the effectiveness of standard-dose DOACs with warfarin in patients with AF and 1 low-risk, non-sex-related stroke risk factor. There was no significant difference observed when comparing rates of stroke/systemic embolism between any of the DOACs (including apixaban) and warfarin. However, it is worth recognizing that apixaban was once again related to lower rates of bleeding $(\mathrm{HR}=0.35 ; 95 \% \mathrm{CI}=0.17-0.72) .{ }^{26}$

These findings were supported by a large retrospective analysis of German patients recruited between January 2013 and March 2015. In total, 835 apixaban and 835 warfarin users were matched using a 1:1 propensity score and there was no difference in rates of ischemic stroke ( $\mathrm{HR}=1.51 ; 95 \%$ $\mathrm{CI}=0.54-4.24)$ and myocardial infarction $(\mathrm{HR}=0.33 ; 95 \%$ $\mathrm{CI}=0.11-1.03$ ) between the 2 cohorts. ${ }^{27}$

A larger study, once again set in Denmark, recruited 61,678 patients from 3 nationwide databases with NVAF who were naïve to anticoagulation, with the aim of assessing the efficacy of anticoagulation by restricting outcomes to ischemic stroke (rather than incorporating hemorrhagic stroke). The study concluded that there was no significant difference in rates of ischemic stroke between DOACs (including apixaban $5 \mathrm{mg}$ bd) and warfarin, however, apixaban was associated with a significantly lower all-cause mortality, which could be explained by the reduced incidence of bleeding events recorded in these patients when compared with warfarin. ${ }^{28}$

\section{Patient outcomes on a reduced dose of apixaban}

The same setup was used in a study that evaluated the efficacy of lower dose DOACs (apixaban $2.5 \mathrm{mg}$ bd, dabigatran $110 \mathrm{mg}$ $\mathrm{bd}$, and rivaroxaban $15 \mathrm{mg}$ od). The evaluation of the Danish database yielded 88,141 patients eligible for inclusion, of whom $69.9 \%$ received warfarin, $7.9 \%$ apixaban, $15.9 \%$ dabigatran, and $6.3 \%$ rivaroxaban. Those treated with apixaban were older, with a mean age of 83.9 years compared with an average age of 73.9 years in the study population, and had more comorbidities such as heart failure, ischemic heart disease, previous ischemic stroke, vascular disease and diabetes mellitus, which ultimately resulted in a greater $\mathrm{CHA}_{2} \mathrm{DS}_{2}$-VASC (congestive cardiac failure, hypertension, age $\geq 75$ years old, diabetes mellitus, stroke/ transient ischemic attack, vascular disease, age between 65-74 years old, female sex category) score of 4.3 , compared with the overall average of 3.3. Patients in the apixaban treatment group also had an increased (but less pronounced) risk of bleeding, summarized as a mean HAS-BLED score of 2.8 compared with an overall average score of $2.4 .^{29}$

Apixaban was associated with the poorest outcomes, while dabigatran and rivaroxaban had a non-significant trend toward lower rates of stroke/systemic embolism. The weighted event rates for bleeding outcomes were similar for apixaban, rivaroxaban, and warfarin at 5.1\%,5.6\%, and 5.1\%, respectively, and lower for dabigatran (4.1\%), while there was no significant difference when apixaban was compared with warfarin (Table 3). ${ }^{29}$

A reduced dose of apixaban is indicated in patients with at least 2 of the following: age $\geq 80$ years, body weight $\leq 60 \mathrm{~kg}$, and serum creatinine $\geq 132.6 \mu \mathrm{mol} / \mathrm{L}$. This study raises concerns 
Table 3 Summary of studies comparing low dose apixaban with warfarin

\begin{tabular}{|c|c|c|c|}
\hline Study & Number of participants & Outcomes & Hazard ratio $(95 \% \mathrm{CI})$ \\
\hline Nielsen et $\mathrm{al}^{29}$ & $88,|4|$ & Stroke/systemic embolism at I year & $1.19(0.95-1.49)$ \\
\hline Comparison of low-dose apixaban and & & Stroke/systemic embolism at 2.5 years & $1.22(1.00-1.50)$ \\
\hline \multirow[t]{5}{*}{ warfarin from a Danish database } & & All-cause mortality at I year & $\mathrm{I} .48(\mathrm{I} .3 \mathrm{I}-\mathrm{I} .67)$ \\
\hline & & All-cause mortality at 2.5 years & $\mathrm{I} .55(\mathrm{I} .39-\mathrm{I} .74)$ \\
\hline & & Any bleeding & $0.96(0.73-1.27)$ \\
\hline & & Major bleeding & $\mathrm{I} .04(0.76-\mathrm{I} .43)$ \\
\hline & & Hemorrhagic stroke & $0.59(0.34-1.02)$ \\
\hline Halvorsen et $\mathrm{al}^{30}$ & 790 & Stroke/systemic embolism & $0.52(0.25-1.08)$ \\
\hline $\begin{array}{l}\text { Post hoc analysis of the ARISTOTLE trial } \\
\text { comparing low-dose apixaban to warfarin }\end{array}$ & & Major bleeding & $0.55(0.31-0.94)$ \\
\hline in patients $\geq 75$ years old & & & \\
\hline
\end{tabular}

over prescribing a reduced dose due to poorer outcomes that are not evident when the standard dose is prescribed. However, the interpretation of these results is limited due to the apixaban cohort already having an increased baseline risk of stroke due to an increased number of comorbidities. ${ }^{29}$

The main clinical trial used to assess the efficacy of reduced-dose apixaban was a post hoc analysis on the ARISTOTLE trial, which demonstrated non-significant trend toward lower rates of stroke/systemic embolism and significant reduction in major bleeding compared with warfarin (Table 3). ${ }^{30}$

The results observed in this trial are in stark contrast to those observed when analyzing real-world data, where reduced-dose apixaban resulted in higher rates of ischemic stroke, without reducing the risk of bleeding. These discrepancies could be due to various factors. First, as aforementioned, the apixaban cohort within the real-world data was already at a higher risk of stroke prior to treatment. Second, given that in healthy people (with normal renal function and a lower age), reduced-dose apixaban was associated with $50 \%$ lower plasma concentrations than treatment with standard dose; inappropriate prescribing patterns of reduced-dose apixaban in clinical practice would lead to sub-therapeutic plasma levels, and render those individuals at a higher risk of thromboembolic events. However, if the reduced dose is being utilized appropriately in clinical practice, these observational data raise the question as to whether a dose reduction of $50 \%$ in patients that meet the afore-listed criteria, is potentially too excessive to maintain effective stroke prevention.

This notion of clinicians inappropriately reducing the dose of apixaban has been investigated by retrospectively reviewing 224 hospitalized patients administered a DOAC at a reduced dose between January 2011 and August 2014. The study found that only $43.3 \%$ of patients fit criteria for a dose adjustment according to manufacturer recommendations, and specifically only 3 of $24(12.5 \%)$ patients treated with apixaban met 2 out of the 3 clinical criteria (outlined previously) required for a dose reduction, while 8 of 24 patients (33.3\%) met none of these criteria, and 54.2\% (13/24) met only 1 of the 3 criteria. $^{31}$

The analysis concluded that the decision to reduce the dose of apixaban was influenced by mild/moderate renal dysfunction, previous hemorrhage, and concomitant use of medications that increased bleeding risk, rather than the recommendations outlined by the manufacturer. ${ }^{31}$ Deviations from such guidelines may go some way to explaining the worse outcomes seen when the apixaban dose is reduced in clinical practice.

These findings were further supported by a retrospective analysis of a USA database, used to investigate how the presence of chronic kidney disease affected prescribing practices. The study identified that of 1473 patients with a renal indication for dose reduction, $43.0 \%$ were potentially overdosed, while of 13,392 patients with no renal indication for dose reduction, $13.3 \%$ were potentially underdosed, and importantly this underdosing was associated with a higher risk of stroke in those treated with apixaban $(\mathrm{HR}=4.87 ; 95 \%$ $\mathrm{CI}=1.30-18.26$ ), compared with those dosed correctly. No such relationships were identified when assessing rivaroxaban and dabigatran. ${ }^{32}$

Correct dosing of apixaban is of paramount importance. While dose reduction may be necessary in certain patients, it must only be used in accordance with the guidelines, otherwise the stroke risk is increased, without significantly reducing the bleeding risk. This was confirmed by retrospective analysis of 17,322 patients from the ARISTOTLE trial who possessed 1 of the 3 dose-reduction criteria and were, therefore, eligible for standard-dose apixaban. These patients had higher rates of stroke/systemic embolism ( $\mathrm{HR}=1.47 ; 95 \%$ $\mathrm{CI}=1.20-1.81)$ and major bleeding $(\mathrm{HR}=1.89$; 95\% $\mathrm{CI}=1.62-$ $2.20)$ than the 13,356 patients with no dose-reduction criteria, 
which could be explained by their inherently higher risk of both thromboembolism and bleeding. ${ }^{33}$ When 8665 patients with 1 dose-reduction criterion treated with apixaban were compared with 8657 matched patients treated with warfarin, there were similar rates of stroke/systemic embolism ( $\mathrm{HR}=0.94 ; 95 \% \mathrm{CI}=0.66-1.32)$ but reduced rates of bleeding $(\mathrm{HR}=0.68 ; 95 \% \mathrm{CI}=0.53-0.87)$. These patterns were consistent with each dose-reduction criterion and across the spectrum of age, body weight, and renal function, and therefore, in patients with an isolated criterion for dose reduction, a standard-dose regimen should be implemented to maintain sufficient stroke prophylaxis, without increasing the bleeding risk. ${ }^{33}$

\section{Patient outcomes of apixaban reflected in its cost effectiveness}

The promising results of standard-dose apixaban were reflected in a further retrospective propensity-matched study that evaluated not only the risk for major bleeding, but also the subsequent risk of hospitalization, and healthcare cost of such complications. During the follow-up, risks for all-cause hospitalizations were greater in rivaroxaban $(\mathrm{HR}=1.44 ; 95 \%$ $\mathrm{CI}=1.2-1.7)$, and dabigatran $(\mathrm{HR}=1.98 ; 95 \% \mathrm{CI}=1.6-2.4)$ than apixaban. This was also reflected in a significantly greater adjusted total all-cause healthcare cost (defined as the total cost of inpatient admissions, outpatient medical services and pharmacy claims) when rivaroxaban was compared with apixaban ( $\$ 4333$ vs $\$ 3950$ per patient per month [PPPM]; $P=0.002$ ), but there was no significant difference when dabigatran was compared with apixaban, despite a lower risk of all-cause hospitalization. The economic benefit of apixaban was further supported when its use was compared with warfarin, and demonstrated reduced total all-cause healthcare costs ( $\$ 3919$ vs $\$ 4177$ PPPM; $P=0.025$ ) and major bleeding-related medical costs ( $\$ 96$ vs $\$ 212$ PPPM; $P=0.026) .{ }^{34}$

The cost effectiveness of apixaban was supported by a simulation study of 1000 patients, over a lifetime horizon with NVAF in a Greek healthcare setting. Apixaban was shown to result in 26 fewer strokes/systemic embolisms in total, 65 fewer bleeds, 41 fewer myocardial infarctions, and 29 fewer cardiovascular-related deaths, with an incremental cost-effectiveness ratio (ICER) of $€ 14,478$ /quality-adjusted life-year (QALY) when compared with warfarin. In patients deemed unsuitable for treatment with warfarin, apixaban was compared with aspirin and resulted in 72 fewer strokes/ systemic embolisms and 57 fewer cardiovascular-related deaths, with an ICER of $€ 7104 /$ QALY. ${ }^{35}$
A study based in the USA constructed a Markov statetransition model to evaluate lifetime costs and QALYs, and assessed the cost effectiveness of 6 forms of anticoagulation (apixaban $5 \mathrm{mg}$ bd, edoxaban $60 \mathrm{mg}$ od, dabigatran $150 \mathrm{mg}$ bd, dabigatran $110 \mathrm{mg}$ bd, rivaroxaban $20 \mathrm{mg}$ od, warfarin) in patients deemed as having a high bleeding risk (HASBLED score $\geq 3$ ). The study concluded that for patients with a creatinine clearance $>95 \mathrm{~mL} / \mathrm{min}$, apixaban $5 \mathrm{mg}$ was the most cost-effective treatment for willingness-to-pay (WTP) thresholds $>\$ 80,000 /$ QALY gained, and in those with creatinine-clearance between $50-95 \mathrm{~mL} / \mathrm{min}$, apixaban $5 \mathrm{mg}$ had an even greater cost-effectiveness with WTP thresholds $>\$ 115,000 /$ QALY gained. $^{36}$

A large systematic review identified 7 Phase II and 16 Phase III randomized controlled trials that sought to evaluate the effectiveness of various forms of stroke prophylaxis in NVAF. The review aimed to determine the best oral anticoagulant in terms of efficacy, safety and cost effectiveness. The trials were conducted worldwide and all but 1 were multicenter, with the total of 94,656 patients being examined. Thirteen of the studies assessed DOACs, 3 of which evaluated apixaban. ${ }^{37}$

The review concluded that all DOACs (apixaban $5 \mathrm{mg}$ bd, dabigatran $150 \mathrm{mg}$ bd, edoxaban $60 \mathrm{mg}$ od, rivaroxaban 20 mg od) were more clinically effective than warfarin, and that apixaban was ranked the best for a wide range of outcomes, including stroke, systemic embolism, major bleeding and all-cause mortality. ${ }^{37}$

This was reflected in the subsequent cost-effectiveness analysis where apixaban was found to have the highest expected QALY, and at a WTP threshold of $£ 20,000 /$ QALY, apixaban had the highest expected incremental net benefit (INB) of $£ 7533$, compared with dabigatran ( $£ 6565$ ), rivaroxaban (£5279) and edoxaban (£5212); and was the only DOAC with a statistically significant INB when compared with warfarin, thought to be due to lower rates of myocardial infarction, intracranial hemorrhage and clinically relevant bleeding. ${ }^{37}$

Despite this, DOAC use is not proportional to the overwhelming success in clinical trials. In a small retrospective trial of 180 patients with AF, only $19(15.1 \%)$ were prescribed DOACs (10 apixaban), compared with 106 on warfarin, despite $37(34.6 \%)$ of these patients having a TTR $<65 \%$, and therefore, being unsuitable for warfarin therapy. ${ }^{38}$

These prescribing practices have been reflected in the larger PREFER in AF study, which enrolled 7 European countries and registered 7243 patients diagnosed with AF. It 
demonstrated that $66.3 \%$ of patients were treated with VKAs compared with only $6.1 \%$ with DOACs, and of those treated with DOACs, apixaban $(0.1 \%)$ was the least popular when compared with dabigatran $(4.0 \%)$ and rivaroxaban $(1.9 \%) .{ }^{39}$

These results are staggering when we consider not only the overall benefit of apixaban when compared with warfarin and other DOACs, but also its greater economic advantages. We can only speculate as to why this may be the case. Clinicians may be restricted by clinical guidelines or influenced by patient preference, while some may not be fully aware of the aforementioned cost benefits, as the initial prescription of apixaban is still more expensive than warfarin. Furthermore, warfarin has been the drug of choice for many years, and certain clinicians may feel a greater sense of familiarity when prescribing it. Prescribing practices must be reevaluated, with newer therapies offering efficacy, safety and convenience as well as (apixaban, in particular) offering far better cost effectiveness than warfarin. ${ }^{40}$

\section{Future perspectives}

Impact of apixaban on patients with subclinical NVAF Apixaban has clear, documented evidence to support its use in patients with paroxysmal, persistent or permanent NVAF to prevent thromboembolic strokes. However, a subset of patients also exists, who have device-detected subclinical AF, which is defined as infrequent, short-lasting, asymptomatic AF detected only with long-term continuous monitoring. Subclinical AF is thought to be common and associated with an increased risk of stroke, but thus far, this subset of patients has not been extensively studied and the net benefit of anticoagulation is unknown. ${ }^{41}$

The Apixaban for the Reduction of Thrombo-Embolism in Patients With Device-Detected Sub-Clinical AF trial is a prospective, multicenter, double-blind, randomized controlled trial, that will enroll $\sim 4000$ patients from 230 sites with subclinical AF, and compare the efficacy of apixaban (dosed according to guidelines) against aspirin (81 mg od), in the prevention of stroke, TIA with diffusion-weighted magnetic resonance imaging evidence of cerebral infarction, and systemic embolism. If this trial yields positive results, the use of apixaban could expand even further to this subset of patients and potentially improve their long-term outcomes. ${ }^{42}$

\section{Impact of apixaban on direct current (DC)-} cardioversion outcomes in NVAF

Another growing use of apixaban is in stroke prophylaxis prior and post, elective DC-cardioversion in patients with NVAF. DC-cardioversion carries a significant risk of ischemic stroke, occurring at a rate of 5\%-7\%. This risk is still significant up to 4 weeks post restoration of sinus rhythm, due to a phenomenon known as "atrial stunning", which describes atrial dysfunction post DC-cardioversion. Therefore, anticoagulation must be continued during this period. ${ }^{43,44}$ Currently, VKAs are the only licensed pre- and post-procedural anticoagulation agents (unless the onset of AF is $<48$ hours prior to DC-cardioversion, in which case, heparin can be used). However, more recently due to convenience, apixaban has begun to be utilized. ${ }^{45}$

In the ARISTOTLE trial a total of 743 DC-cardioversions were performed in 540 patients (265 in the apixaban cohort and 275 in the warfarin cohort). Baseline characteristics were similar between both groups and none had any evidence of a thrombus in the left atrial appendage seen on a transoesophageal echocardiogram prior to DC-cardioversion. Follow-up was 30 days after the procedure. During this time, no patients had suffered from stroke/systemic embolism, but 1 patient in each cohort had a myocardial infarction and 1 a major bleeding episode. It was, therefore, concluded that apixaban offered a safe and useful alternative to warfarin. ${ }^{46}$

\section{Impact of apixaban on elective ablation outcomes in NVAF}

The successful use of apixaban in elective DC-cardioversion has also led to apixaban being used as stroke prophylaxis prior to radiofrequency catheter ablation, in patients with NVAF. The rationale behind restoration of sinus rhythm is to improve survival, lessen the risk of thromboembolism, eliminate the necessity for oral anticoagulation, preserve atrial contraction and improve cardiac output. ${ }^{47}$ However, anticoagulation is still required prior to the procedure due to the ongoing thromboembolic risk, and although only a low stroke risk has been reported following the procedure, anticoagulation is recommended for at least 3 months after. ${ }^{45}$

The efficacy and safety of uninterrupted apixaban therapy prior to radiofrequency ablation was evaluated by comparing patient outcomes to those anticoagulated with warfarin (currently the gold standard). A total of 627 patients underwent the procedure between January 2013 and February 2016. Of the 317 patients in the apixaban group, only 5 had bleeding complications compared with 8 out of the 310 patients in the warfarin group. There were no thromboembolic complications in either group. ${ }^{48}$

Retrospective analysis of 342 consecutive patients who underwent radiofrequency ablation for AF between April 2013 and March 2014 in Japan, also revealed no significant differences in both thromboembolic and bleeding 
complications between the 105 patients in the apixaban group and 237 in the warfarin group. ${ }^{49}$

A similar prospective multicenter trial across 4 institutions in USA and Europe confirmed the aforementioned findings. A total of 400 patients were recruited, with 200 randomized to each cohort. There was no significant difference with regard to the total bleeding complications between the apixaban and warfarin groups $(4.5 \%$ vs $3 \% ; P=0.43)$, and once again, no thromboembolic complications were recorded. Furthermore, all cerebral diffusion-weighted magnetic resonance imaging of those in the apixaban group were negative for "new" silent cerebral ischemic events. ${ }^{50}$

Thus, it has been concluded that use of uninterrupted apixaban administration in patients undergoing radiofrequency ablation seems to be a feasible and effective alternative to warfarin in preventing clinical and silent thromboembolic events without increasing the risk of major bleeding.

\section{Apixaban reversal and the potential to reduce hemorrhagic complications}

Until recently, the main fear of initiating therapy with a DOAC was the lack of reversibility, which the older longer acting VKAs do offer. This was an issue until the reversal agent, idarucizumab, was proven to completely reverse dabigatran. ${ }^{51}$ Some would argue that given apixaban has a shorter half-life and better safety profile than the other DOACs, its need for a reversal agent is also less. In spite of this, adexanet alfa has recently been developed and is a recombinant mimetic of human factor Xa that binds factor Xa inhibitors but does not have any intrinsic catalytic activity. Adexanet alfa administration achieved good hemostasis in 75\% $(95 \%$ $\mathrm{CI}=51 \%-91 \%$ ) of bleeding patients taking apixaban. After a bolus administration, the median anti-factor Xa activity decreased by $93 \%(95 \% \mathrm{CI}=87 \%-94 \%)$ and these levels remained similar during the 2 -hour infusion. There were no transfusion reactions, which rendered adexanet alfa a safe, rapid and effective apixaban reversal agent. ${ }^{52}$

Adexanet alfa is still in the early stages of its development; with trials still ongoing and further evidence is required before its use becomes widespread. Early results are promising, and its emergence may counter clinicians' fears regarding reversibility and also improve patient outcomes further by reducing the morbidity and mortality from any apixabanrelated bleeding complications.

\section{Conclusion}

Apixaban is an effective and safe form of anticoagulation in patients with NVAF. It successfully reduces the rate of thromboembolic complications, while also carrying a lower risk of bleeding than its counterparts. When a standard dose is prescribed, it is just as effective as the other DOACs and warfarin in preventing ischemic strokes, and even more effective than its equivalents in preventing hemorrhagic strokes. The reduced incidence of hemorrhagic strokes may be, in part, due to its impressive safety profile. Apixaban has been associated with reduced rates of gastrointestinal and intracranial bleeding, and this has also been reflected in reduced mortality rates. Concerns, however, have been raised with the reduced dosing regimen, which has not echoed the same impressive results. However, this is partly explained by clinicians not adhering to the guidelines, and prescribing this regimen in patients who should be receiving the standard dose, and therefore, undertreating them and unintentionally increasing their risk of thromboembolic complications. The general success of apixaban in improving patient outcomes has been reflected in its cost effectiveness, which has been echoed worldwide. Along with its exponentially growing number of applications related to AF (such as anticoagulation prior to DC-cardioversion and radiofrequency ablation), and newly developed reversal agent, the use of apixaban as a form of stroke prophylaxis in patients with NVAF is likely to increase further, and continue to improve patient outcomes.

\section{Disclosure}

The authors report no conflicts of interest in this work.

\section{References}

1. Lip GYH, Brechin CM, Lane DA. The global burden of atrial fibrillation and stroke: a systematic review of the epidemiology of atrial fibrillation in regions outside North America and Europe. Chest. 2012;142(6):1489-1498.

2. Chugh SS, Havmoeller R, Narayanan K, et al. Worldwide epidemiology of atrial fibrillation: a Global Burden of Disease 2010 Study. Circulation. 2014;129(8):837-847.

3. Lip GY, Tse HF, Lane DA. Atrial fibrillation. Lancet. 2012;379(9816): 648-661.

4. Morgan CL, McEwan P, Tukiendorf A, Robinson PA, Clemens A, Plumb JM. Warfarin treatment in patients with AF: observing outcomes associated with varying levels of INR control. Thromb Res. 2009;124(1):37-41.

5. Reynolds MW, Fahrbach K, Hauch O, et al. Warfarin anticoagulation and outcomes in patients with AF: a systematic review and metaanalysis. Chest. 2004;126(6):1938-1945.

6. Raghavan N, Frost CE, Yu Z, et al. Apixaban metabolism and pharmacokinetics after oral administration to humans. Drug Metab Dispos. 2009;37(1):74-81.

7. Hart RG, Pearce LA, Aguilar MI. Meta-analysis: antithrombotic therapy to prevent stroke in patients who have nonvalvular AF. Ann Intern Med. 2007;146(12):857-867.

8. Connolly SJ, Eikelboom J, Joyner C, et al; AVERROES Steering Committee and Investigators. Apixaban in patients with atrial fibrillation. N Engl J Med. 2011;364(9):806-817. 
9. Granger CB, Alexander JH, McMurray JJ, et al; ARISTOTLE Investigators. Apixaban versus warfarin in patients with AF. $N$ Engl J Med. 2011;365(11):981-992.

10. Easton JD, Lopes RD, Bahit MC, et al; ARISTOTLE Committees and Investigators. Apixaban compared with warfarin in patients with atrial fibrillation and previous stroke or transient ischaemic attack: a subgroup analysis of the ARISTOTLE trial. Lancet Neurol. 2012;11(6):503-511.

11. McMurray JJ, Ezekowitz JA, Lewis BS et al; ARISTOTLE Committees and Investigators. Left ventricular systolic dysfunction, heart failure, and the risk of stroke and systemic embolism in patients with atrial fibrillation: insights from the ARISTOTLE trial. Circ Heart Fail. 2013;6(3):451-460.

12. Bahit MC, Lopes RD, Wojdyla DM, et al. Apixaban in patients with atrial fibrillation and prior coronary artery disease: insights from the ARISTOTLE trial. Int J Cardiol. 2013;170(2):215-220.

13. Hu PT, Lopes RD, Stevens SR, et al. Efficacy and safety of apixaban compared with warfarin in patients with atrial fibrillation and peripheral artery disease: insights from the ARISTOTLE trial. JAm Heart Assoc. 2017;6(1):e004699.

14. Hohnloser SH, Hijazi Z, Thomas L, et al. Efficacy of apixaban when compared with warfarin in relation to renal function in patients with atrial fibrillation: insights from the ARISTOTLE trial. Eur Heart $J$. 2012;33(22):2821-2830.

15. Ezekowitz JA, Lewis BS, Lopes RD, et al; Clinical outcomes of patients with diabetes and atrial fibrillation treated with apixaban: results from the ARISTOTLE trial. Eur Heart $J$ Cardiovasc Pharmacother. 2015;1(2):86-94.

16. Jaspers Focks J, Brouwer MA, Wojdyla DM, et al. Polypharmacy and effects of apixaban versus warfarin in patients with atrial fibrillation: post hoc analysis of the ARISTOTLE trial. BMJ. 2016;353:i2868.

17. Rao MP, Halvorsen S, Wojdyla D, et al; ARISTOTLE Committee and Investigators. Blood pressure control and risk of stroke or systemic embolism in patients with atrial fibrillation: results from the apixaban for reduction in stroke and other thromboembolic events in atrial fibrillation (ARISTOTLE) trial. J Am Heart Assoc. 2015;4(12):e002015.

18. Garcia DA, Wallentin L, Lopes RD, et al. Apixaban versus warfarin in patients with atrial fibrillation according to prior warfarin use: results from the apixaban for reduction in stroke and other thromboembolic events in atrial fibrillation trial. Am Heart J. 2013;166(3):549-558.

19. Coleman CI, Peacock WF, Bunz TJ, Alberts MJ. Effectiveness and safety of apixaban, dabigatran, and rivaroxaban versus warfarin in patients with nonvalvular AF and previous stroke or transient ischemic attack. Stroke. 2017;48(8):2142-2149.

20. Deitelzweig S, Farmer C, Luo X, et al. Risk of major bleeding in patients with non-valvular AF treated with oral anticoagulants: a systematic review of real-world observational studies. Curr Med Res Opin. 2017;23(9):1583-1594.

21. Lip GY, Keshishian A, Kamble S, et al. Real-world comparison of major bleeding risk among non-valvular atrial fibrillation patients initiated on apixaban, dabigatran, rivaroxaban, or warfarin. A propensity score matched analysis. Thromb Haemost. 2016;116(5):975-986.

22. Xu WW, Hu SJ, Wu T. Risk analysis of new oral anticoagulants for gastrointestinal bleeding and intracranial hemorrhage in AF patients: a systematic review and network meta-analysis. J Zhejiang Univ Sci B. 2017;18(7):567-576.

23. Yao X, Abraham NS, Sangaralingham LR, et al. Effectiveness and safety of dabigatran, rivaroxaban, and apixaban versus warfarin in nonvalvular AF. J Am Heart Assoc. 2016;5:e003725.

24. Amin A, Keshishian A, Trocio J, et al. Risk of stroke/systemic embolism, major bleeding and associated costs in non-valvular AF patients who initiated apixaban, dabigatran, or rivaroxaban compared with warfarin in the United States Medicare population. Curr Med Res Opin. 2017;33(9):1595-1604.

25. Tawfik A, Bielecki JM, Krahn M, et al. Systematic review and network meta-analysis of stroke prevention treatments in patients with AF. Clin Pharmacol. 2016;8:93-107.
26. Lip GYH, Skjøth F, Nielsen PB, Kjældgaard JN, Larsen TB. Effectiveness and safety of standard-dose nonvitamin $\mathrm{K}$ antagonist oral anticoagulants and warfarin among patients with $\mathrm{AF}$ with a single stroke risk factor: A Nationwide Cohort Study. JAMA Cardiol. 2017;2(8):872-881.

27. Coleman CI, Peacock WF, Antz M. Comparative effectiveness and safety of apixaban and vitamin $\mathrm{K}$ antagonist therapy in patients with nonvalvular atrial fibrillation treated in routine German practice. Heart Lung Circ. Epub 2017 May 3.

28. Larsen TB, Skjøth F, Nielsen PB, Kjældgaard JN, Lip GY. Comparative effectiveness and safety of non-vitamin $\mathrm{K}$ antagonist oral anticoagulants and warfarin in patients with AF: propensity weighted nationwide cohort study. BMJ. 2016;353:i3189.

29. Nielsen PB, Skjøth F, Søgaard M, Kjældgaard JN, Lip GY, Larsen TB. Effectiveness and safety of reduced-dose non-vitamin $\mathrm{K}$ antagonist oral anticoagulants and warfarin in patients with AF: propensity weighted nationwide cohort study. BMJ. 2017;356:j510.

30. Halvorsen S, Atar D, Yang H, et al. Efficacy and safety of apixaban compared with warfarin according to age for stroke prevention in AF: observations from the ARISTOTLE trial. Eur Heart J. 2014;35(28):1864-1872.

31. Barra ME, Fanikos J, Connors JM, Sylvester KW, Piazza G, Goldhaber SZ. Evaluation of dose-reduced direct oral anticoagulant therapy. Am J Med. 2016;129(11):1198-1204.

32. Yao X, Shah ND, Sangaralingham LR, Gersh BJ, Noseworthy PA. NonVitamin $\mathrm{K}$ antagonist oral anticoagulant dosing in patients with $\mathrm{AF}$ and renal dysfunction. J Am Coll Cardiol. 2017;69(23):2779-2790.

33. Alexander JH, Andersson U, Lopes RD, et al; ARISTOTLE Investigators. Apixaban $5 \mathrm{mg}$ twice daily and clinical outcomes in patients with AF and advanced age, low body weight, or high creatinine: a secondary analysis of a randomized clinical trial. JAMA Cardiol. 2016;1(6):673-81.

34. Lin J, Trocio J, Gupta K, et al. Major bleeding risk and healthcare economic outcomes of non-valvular AF patients newly-initiated with oral anticoagulant therapy in the real-world setting. J Med Econ. 2017;20(9):952-961.

35. Athanasakis K, Boubouchairopoulou N, Karampli E, et al. Cost effectiveness of apixaban versus warfarin or aspirin for stroke prevention in patients with AF: a Greek perspective. Am J Cardiovasc Drugs. 2017;17(2):123-133.

36. Hernandez I, Smith KJ, Zhang Y. Cost-effectiveness of non-vitamin $\mathrm{K}$ antagonist oral anticoagulants for stroke prevention in patients with $\mathrm{AF}$ at high risk of bleeding and normal kidney function. Thromb Res. 2017;150:123-130.

37. Sterne JA, Bodalia PN, Bryden PA, et al. Oral anticoagulants for primary prevention, treatment and secondary prevention of venous thromboembolic disease, and for prevention of stroke in AF: systematic review, network meta-analysis and cost-effectiveness analysis. Health Technol Assess. 2017;21(9):1-386.

38. Ioannou A, Metaxa S, Kassianos G, Missouris CG. Anticoagulation for the prevention of stroke in non-valvular AF in general practice: room for improvement. Drugs Context. 2016;5:212295.

39. Kirchhof P, Ammentorp B, Darius H, et al. Management of AF in seven European countries after the publication of the 2010 ESC Guidelines on atrial fibrillation: primary results of the PREvention oF thromboemolic events European Registry in AF (PREFER in AF). Europace. 2014;16(1):6-14.

40. Kasmeridis C, Apostolakis S, Ehlers L, Rasmussen LH, Boriani G, Lip GY. Cost effectiveness of treatments for stroke prevention in AF: focus on the novel oral anticoagulants. Pharmacoeconomics. 2013;31(11):971-980.

41. Healey JS, Connolly SJ, Gold MR, et al; ASSERT Investigators. Subclinical AF and the risk of stroke. N Engl J Med. 2012;366(2):120-129.

42. Lopes RD, Alings M, Connolly SJ, et al. Rationale and design of the apixaban for the reduction of thrombo-embolism in patients with devicedetected sub-clinical AF (ARTESiA) trial. Am Heart J. 2017;189:137-145.

43. Lip GY, Gitt AK, Le Heuzey JY, et al. Overtreatment and undertreatment with anticoagulation in relation to cardioversion of atrial fibrillation (the RHYTHM-AF study). Am J Cardiol. 2014;113(3):480-484. 
44. Khan IA. Atrial stunning: basics and clinical considerations. Int $J$ Cardiol. 2003;92(2-3):113-128.

45. European Heart Rhythm Association; European Association for CardioThoracic Surgery. Camm AJ, Kirchhof P, Lip GY, et al. Guidelines for the management of AF: the task force for the management of AF of the European Society of Cardiology (ESC). Eur Heart J. 2010;31(19):2369-2429.

46. Flaker G, Lopes RD, Al-Khatib SM, et al; ARISTOTLE Investigators. Efficacy and safety of apixaban in patients after cardioversion for AF: insights from the ARISTOTLE Trial (Apixaban for Reduction in Stroke and Other Thromboembolic Events in AF). J Am Coll Cardiol. 2014;63(11):1082-1087.

47. Funatsu T, Kobayashi J, Nakajima H, Iba Y, Shimahara Y, Yagihara T. Long-term results and reliability of cryothermic ablation based maze procedure for atrial fibrillation concomitant with mitral valve surgery. Eur J Cardiothorac Surg. 2009;36(2):267-271.
48. Shah RR, Pillai A, Schafer P, et al. Safety and efficacy of uninterrupted apixaban therapy versus warfarin during AF ablation. Am J Cardiol. 2017;120(3):404-407.

49. Nagao T, Inden $Y$, Shimano M, et al. Efficacy and safety of apixaban in the patients undergoing the ablation of atrial fibrillation. Pacing Clin Electrophysiol. 2015;38(2):155-163.

50. Di Biase L, Lakkireddy D, Trivedi C, et al. Feasibility and safety of uninterrupted periprocedural apixaban administration in patients undergoing radiofrequency catheter ablation for AF: results from a multicenter study. Heart Rhythm. 2015;12(6):1162-1168.

51. Pollack CV Jr, Reilly PA, Eikelboom J, et al. Idarucizumab for dabigatran reversal. N Engl J Med. 2015;373(6):511-520.

52. Connolly SJ, Milling TJ Jr, Eikelboom JW, et al; ANNEXA-4 Investigators. Andexanet alfa for acute major bleeding associated with factor Xa inhibitors. N Engl J Med. 2016;375(12):1131-1141.
Patient Related Outcome Measures

\section{Publish your work in this journal}

Patient Related Outcome Measures is an international, peer-reviewed, open access journal focusing on treatment outcomes specifically relevant to patients. All aspects of patient care are addressed within the journal and practitioners from all disciplines are invited to submit their work as well as healthcare researchers and patient support groups.

\section{Dovepress}

The journal is included in PubMed. The manuscript management system is completely online and includes a very quick and fair peer-review system. Visit http://www.dovepress.com/testimonials.php to read real quotes from published authors.

Submit your manuscript here: http://www.dovepress.com/patient-related-outcome-measures-journal 